\title{
Análise de Hospitalidade e Gastronomia em Puerto Vallarta, Jalisco, México
}

\author{
Analysis of Hospitality and Gastronomy in Puerto Vallarta, Jalisco, Mexico
}

\author{
Carlos Rogelio Virgen Aguilar \\ Lorena Trinidad Medina Esparza \\ Ángel Gabriel de Niz Sedano
}

Centro Universitario de la Costa - Universidad de Guadalajara - Puerto Vallarta - México

Resumo: Puerto Vallarta, Jalisco, é um destino turístico no Pacífico mexicano que fez do turismo um dos principais fatores para o desenvolvimento econômico, estando entre os três primeiros lugares no México em termos de receber visitantes, não há uma concentração excessiva de atividade econômica em torno do turismo, já que cerca de $80 \%$ dos empregos no Porto estão relacionados a atividades relacionadas a ele. Em 2011, mais de $80 \%$ da população empregada de Puerto Vallarta estava trabalhando no setor terciário, neste quadro que institui a percepção da comunidade local no que diz respeito ao turismo, uma importante hospitalidade mesmo acordo quadrantes elemento que poderia ser estabelecido a partir da matriz de Bjorklund e Philbrick, vale ressaltar que $69,5 \%$ dos habitantes pesquisados têm uma aceitação aberta a essa atividade, $24 \%$ têm uma aceitação em silêncio e apenas 2,6\% manifestam uma rejeição aberta. Em termos de empresas de alimentos de alimentos e bebidas, totalizando 1.645 unidades econômicas acordo com as estatísticas National Board (DENUE, 2017) impressão de valor considerável na indústria de alimentos para o destino, depois de analisar a classificação das empresas de alimentos é notável domínio rua barracas de comida expressa em tacos com $34,2 \%$ dos estabelecimentos deste tipo, contra $23,8 \%$ dos estabelecimentos restaurantes à la carte.

Palavras-chaves: Hospitalidade, gastronomia, Puerto Vallarta

\begin{abstract}
Puerto Vallarta, Jalisco, is a tourist destination of the Mexican Pacific that has made tourism one of the main factors for economic development, ranking among the first three places in Mexico in terms of reception of visitors, there being an excessive concentration of economic activity around tourism, since close to $80 \%$ of jobs in the Port are related to activities related to it. In 2011, more than $80 \%$ of the employed population of Puerto Vallarta worked in the tertiary sector, in this framework establishing the perception that the local community has regarding tourism, an important element in the hospitality of the same agreement to the quadrants that could be established from the matrix of Bjorklund and Philbrick, it is noteworthy that $69.5 \%$ of the surveyed inhabitants have an open acceptance to this activity, $24 \%$ have an acceptance in silence and only $2.6 \%$ manifest an open rejection. In terms of gastronomy, food and beverages companies total a total of 1,645 Economic Units according to the National Statistical Directorate (DENUE, 2017) printing a considerable value of the gastronomic sector to the destination, after analyzing the classification of food companies is notable the predominance of street food expressed in taco stands with $34.2 \%$ of establishments of this type, against $23.8 \%$ of establishments of a la carte restaurants.
\end{abstract}

Keywords: Hospitality, gastronomy, Puerto Vallarta 


\section{Introducción}

Unos de los aspectos importantes en el estudio de la actividad turística lo constituye el análisis de la oferta, los impactos que genera en la comunidad receptora y el nivel de aceptación de ésta. En este artículo se analiza la evolución de Puerto Vallarta como destino turístico, de su oferta de hospedaje, la demanda, y los impactos desde los puntos de vista económico, social y ambiental, así como el nivel de aceptación del turismo de parte de los habitantes (hospitalidad).

Finalmente se analiza los establecimientos de alimentos y bebidas y su distribución en la estructura del destino.

\section{2 ¿Qué es Puerto Vallarta?}

Puerto Vallarta puede considerarse un destino de litoral, de desarrollo tradicional no planificado, donde la afluencia masiva de turistas ha contribuido en gran medida al crecimiento de la economía local, aunque la consecuencia haya sido la transformación de amplias zonas, especialmente desde el punto de vista paisajístico, y una presión, a veces excesiva, sobre los recursos naturales debido a una insuficiente planificación; siguiendo un modelo de desarrollo urbano-turístico, que de acuerdo con su evolución ha llegado a la etapa de madurez, la cual se manifiesta, entre otros factores, a través del estancamiento de la oferta turística, el deterioro del espacio turístico, un menor crecimiento relativo de los segmentos de demanda con mayor valor añadido y deseado, con respecto a otros destinos que operan en la misma línea de producto-mercado.

\section{Antecedentes}

Puerto Vallarta es la segunda zona económica más importante del estado de Jalisco y el tercer puerto más importante de México, de manera que, es uno de los lugares más visitados del país. La toponimia del nombre de Puerto Vallarta, es en honor al Lic. Ignacio L. Vallarta, notable jurisconsultor jalisciense y ex gobernador del estado.

La riqueza natural con que cuenta el municipio está representada por 93,468 hectáreas de bosque, la flora es muy variada; hay árbol de chilte, maderas duras como tampicirán y brasil, y maderas como: amapa, primavera, parota, cedro y nogal, apropiadas para la fabricación de muebles. En la franja costera proliferan las palmeras y otros frutales como el mango, guanábano y aguacate. Una de las especies que más ha sufrido por el crecimiento urbano es la palmera de coquito de aceite que crece en una zona muy restringida y debería estar protegida (JALISCO, 2014: 5).

La fauna está integrada por venados, tigrillos, iguanas y aves como: pericos, guacamayas $y$ palomas. Otras aves como el zopilote, la chachalaca y los patos se han ido retirando del área poco a poco. La fauna marina es también abundante y variada; se pesca sierra, dorado, pez vela, pargo, huachinango, garlopa y bonita, y en el estero El Salado, viven caimanes que están en peligro de extinción (JALISCO, 2014: 3).

Por otra parte, el clima es húmedo-tropical y húmedo con una temperatura media de $28^{\circ} \mathrm{C}$, con una máxima en el verano es de $31.6^{\circ} \mathrm{C}$ y mínima de $19.6^{\circ} \mathrm{C}$. La temporada de lluvias se registra entre los meses de junio, julio y agosto, continuando con lluvias aisladas hasta mediados de octubre, contando con una precipitación media de 1,417.5 milímetros. Los vientos dominantes son en dirección al suroeste y no presenta heladas (VIRGEN, 2009: 4).

El municipio de Puerto Vallarta pertenece a la Región Costa Norte, su población es de 255 mil 681 personas (INEGI, 2010), 50.3\% hombres y 49.7\% mujeres, los habitantes del municipio representaban el $3.5 \%$ del total estatal. 
Tabla 1: Evolución de la población Puerto Vallarta (1960-2010).

\begin{tabular}{|c|c|c|}
\hline Año & Población & Tasa media anual de crecimiento \\
\hline 1960 & 15,472 & - \\
\hline 1970 & 35,911 & 13.2 \\
\hline 1980 & 57,028 & 5.9 \\
\hline 1990 & 111,457 & 9.5 \\
\hline 2000 & 183,741 & 6.5 \\
\hline 2005 & 220,368 & 4.0 \\
\hline 2010 & 255,681 & 4.7 \\
\hline
\end{tabular}

Fuente: INEGI: Censos de población y vivienda 1960, 1970, 1980, 1990, 2000, 2010 y Conteo 2005.

El turismo es uno de los principales factores para el desarrollo económico de este municipio. Puerto Vallarta ofrece a sus habitantes y visitantes un buen número de playas que son visitadas cotidianamente. Aquí se pueden admirar gran cantidad de bellezas naturales, monumentos históricos y obras de arte, lo cual atrae a muchos turistas nacionales y extranjeros.

Además de contar con zonas arqueológicas, ecológicas y de montañas con bellos paisajes, lo cual lo hace ampliamente atractivo para esta actividad económica.

Asimismo, construcciones históricas y ampliamente significativas, obras de arte, costumbres, tradiciones y leyendas muy propias de Puerto Vallarta, sin dejar de lado los eventos nacionales e internacionales que aquí se celebran, representan para sus habitantes y turistas un foco de atracción muy interesante, encontrando sitios de interés como: el Malecón, el Centro Ceremonial Ixtapa, los centros de convenciones, Conchas Chinas, el templo de Nuestra Señora de Guadalupe, Boca de Tomatlán, las Playas Gemelas, Boca de Tomates, la Playa de los Muertos, por mencionar algunos.

\section{Evolución del turismo en Puerto Vallarta}

Puerto Vallarta ha sido un destino turístico que ha experimentado una evolución heterogénea a través de su historia, donde se observan periodos de alto y rápido crecimiento y otros en que el ritmo de crecimiento ha bajado, e incluso, en los que se observa una disminución, estos cambios son, en la mayoría de los casos, producto de la economía de los mercados emisores, tanto en el turismo doméstico como en el internacional o de la poca diversificación de éstos.

La trayectoria que ha seguido este destino turístico desde los años cincuenta es la siguiente:

1955-1964: Despegue de la actividad turística en pequeños hoteles localizados en el pueblo de Puerto Vallarta.

1965-1974: Proyección internacional y atracción de un creciente número de turistas.

1975-1984: Rápido crecimiento de la oferta de alojamiento en grandes y modernas instalaciones hoteleras y de unidades de tiempo compartido y tiempo completo; construcción de nuevos equipamientos como la marina y campo de golf. Expansión de la zona hotelera tanto al norte como a sur.

1985-1994: Crecimiento acelerado de la demanda como resultado de la construcción de grandes hoteles y unidades de tiempo compartido. Expansión urbano-turística con una elevada densificación en Puerto Vallarta. Nuevos productos turísticos en Bahía de Banderas que mantienen el modelo de alta densidad y que empiezan a modificar las tendencias de crecimiento de la zona (Nuevo Vallarta y Flamingos). Crisis económica que afectó al turismo nacional y al mercado inmobiliario.

1995-2000: Disminución del ritmo de crecimiento de Puerto Vallarta y consolidación de la tendencia de crecimiento hacia Punta Mixta con nuevos productos de elevada calidad (Punta Mixta y Costa Bandera). Contracción del mercado nacional. Sobreoferta de cuartos, aumento de la participación de 
mayoristas, comercialización de paquetes "Todo Incluido". Recesión económica mundial.

A partir de 2001: Existen importantes proyectos de inversión con el objetivo de mantener el mismo ritmo de crecimiento de la última década, como, por ejemplo, continuar el crecimiento turístico y hotelero posicionando a toda la región de la Bahía de Banderas, a partir de Puerto Vallarta, como un corredor turístico de excelencia (FONATUR, 2001).

Una de las variables más importantes y más utilizada en el análisis del turismo lo constituye la capacidad de hospedaje, ya que a través de éste se mide el potencial real del destino, su evolución y su capacidad para recibir una demanda.

La gran explosión de la capacidad de hospedaje de Puerto Vallarta se registra en el periodo de 1975 a 1990, en que la evolución es espectacular con una tasa anual del 15.2\%, registrándose 1990 como el gran año de la hotelería al crecer en ese año en $21.2 \%$ en relación con el año anterior (VIRGEN, 2014: 60).

Este crecimiento no podría mantenerse, pues si bien fue motivado por una creciente demanda, convirtiéndolo en un mercado de vendedores, donde la demanda superaba a la oferta, el crecimiento de la oferta motivó que se transformara rápidamente en un mercado de compradores, que es cuando la oferta tiende a ser superior a la demanda, provocando así una mayor capacidad de negociación de los demandantes; con la consecuencia de que los precios tienden naturalmente a bajar.

Los oferentes están dispuestos entonces a vender su producto a precios más bajos, dando a veces mejores condiciones de financiamiento y otras facilidades que estimulan las compras; esto se dio con la idea de mantener el crecimiento de la demanda y mantener los coeficientes de ocupación altos, lo cual constituyó un error, pues en el turismo cuando el precio de un servicio baja, es muy difícil volver a recuperar el precio anterior, tal fue el caso de Cancún, que después del huracán Gilberto disminuyeron los precios y tardaron más de 15 años en volver a recuperarlos.

Confirmándose la teoría de que los servicios turísticos son intangibles y altamente perecederos, la producción de éstos no se puede detener, reducir, suspender o almacenar; como ocurriría con otros productos, aumentando con ello la escasez y así se haría subir el precio.

Casi todos los mercados presentan oscilaciones de este tipo, que hacen fluctuar los precios alrededor de algún valor básico, aunque los mercados de ciertos bienes y servicios pueden permanecer, -según sus características específicas-, durante un tiempo relativamente largo en uno $u$ otro extremo. Estas oscilaciones también se presentan, para toda la economía, durante las etapas de expansión y de recesión: en la fase expansiva predominan los mercados de vendedores, mientras que en la contractiva los de compradores.

En el año 2016, Puerto Vallarta contaba con una capacidad de hospedaje de 30,791 de los cuales 8,676 son en establecimientos de 5 estrellas; 7,229 son en establecimientos de 4 estrellas; 2,255 en 3 estrellas; 341 en 2 estrellas; 381 de una estrella y 11,939 son en establecimientos sin categoría. Estos últimos comprenden: hoteles, moteles, cabañas, suites, trailer park, apartamentos, condominios, bungalows, hostales, haciendas, casas, casas rurales, albergues y campamentos (JALISCO, 2016).

Puerto Vallarta es uno de los principales centros turísticos de playa del país; fue despegando paulatinamente en la década de los 70's basado en el modelo del turismo masivo. En la actualidad ese modelo no está completamente en crisis, pero muestra signos de inestabilidad porque han surgido otros nichos de mercado asociados a nuevos patrones de consumo turístico que parecen ofrecer nuevas oportunidades para el desarrollo y el crecimiento de la economía local, altamente dependiente de esta actividad.

Como muchos otros casos en el mundo, Puerto Vallarta enfrenta grandes retos y dificultades para su reestructuración, sobre todo ante la necesidad de emprender la diversificación de su oferta hacia el aprovechamiento y desarrollo de mercados más selectivos y redituables. 
Entre los principales inconvenientes destaca el creciente dominio de las empresas mayoristas trasnacionales, que controlan los flujos de turistas y que imponen pautas empresariales y de desarrollo local, y que han trazado en buena medida el camino y posible destino de este centro turístico; además de nuevas formas de hospedaje.

Igualmente, emergen los problemas de rezago en infraestructura y de marginación social propios de un modelo de desarrollo que privilegia las utilidades económicas. Se trata de una tendencia generalizada en que las determinaciones del desarrollo capitalista muestran el difícil equilibrio entre los intereses empresariales, los requerimientos del sistema turístico, y los imperativos del desarrollo social.

Como un modelo integral, el turismo genera en su desarrollo una serie de impactos muy significativos, que van más allá de las externalidades que estudia la economía y que deben analizarse para tener una base sobre la cual se planteará el desarrollo sustentable.

El turismo es una compleja actividad económica que depende, posiblemente más que las otras, del medio ambiente, pero considerado éste en una perspectiva amplia.

Esto se debe a que el mismo abarca la biosfera, los ecosistemas que la componen y los elementos introducidos en ella por el hombre, contado entre éstos, los aspectos socioeconómicos y culturales.

Este primer acercamiento, lleva a definir tres grandes tipos de impactos: los económicos (que generalmente son los deseados), los socio-culturales y ambientales que son generalmente los no deseados, pero que de cierta manera juegan un papel importante como el trinomio del turismo.

\section{Impactos económicos del turismo en Puerto Vallarta}

El turismo es una de las actividades económicas más importantes a nivel global ya que el papel que tiene en la generación de divisas, de empleos y de inversión lo hace jugar un rol preponderante en las principales economías nacionales. Además, en los últimos años el turismo en el mundo ha crecido a tasas superiores que el crecimiento de la economía en su conjunto.

Esto se debe a la competitividad que existe entre los prestadores de servicios turísticos, por consiguiente, la calidad de los servicios y productos turísticos que éstos ofrecen.

\section{Perfil socioeconómico}

La tercerización de la economía es la transformación de las actividades económicas hacia unas más enfocadas al sector de servicios,

o también llamadas actividades terciarias. Este fenómeno es más notable en las economías desarrolladas o países industrializados en donde la estructura económica ha cambiado hacia una basada fundamentalmente en el sector de servicios en detrimento del sector industrial

La principal causa de la tercerización de la economía ha sido el incremento de la renta de las familias que les ha permitido un mayor consumo de servicios en salud, educación, turismo, hoteleros, seguros, transporte, entre otros. Las familias empiezan a gastar cada vez una mayor proporción de sus ingresos en distintos servicios; mientras que su gasto proporcional en bienes primarios e industriales decrece; así esta reestructuración del presupuesto de las familias es reflejada en el cambio de la producción de la economía, favoreciendo el sector de servicios.

Éste no es un fenómeno reciente, pues inició desde la última fase de la Revolución Industrial, a inicios del siglo $X X$, y se ha intensificado en décadas recientes como consecuencia de la globalización que ha impulsado el desplazamiento de los centros industriales de los países desarrollados a países en desarrollo.

La tercerización empezó en Puerto Vallarta a mediados de los años 50's, cuando los habitantes de este destino empezaron a dejar sus principales actividades económicas como la ganadería, la agricultura y la pesca para entrar a actividades de 
servicio y comercio, principalmente el turismo, ya que éste generaba mayor número de ingresos y así mismo, era el principal generador de empleos en la comunidad.

Tabla 2: Evolución demográfica de Puerto Vallarta hacia la tercerización. Evolución demográfica y población económicamente activa por sector en el municipio de Puerto Vallarta, 1955-2005

\begin{tabular}{llllll}
\hline & \multicolumn{5}{c}{ PEA (\%) } \\
\cline { 2 - 6 } Año & Habitantes & Primario & Secundario & Terciario & No especificado \\
\hline 1955 & 12731 & 67.6 & 14.3 & 8.1 & 10.0 \\
1960 & 15472 & 60.48 & 13.8 & 24.1 & 1.62 \\
1970 & 35911 & 24.7 & 21.6 & 47.6 & 5.8 \\
1980 & 57028 & 11.3 & 15.9 & 38.4 & 34.3 \\
1990 & 111457 & 5 & 15.6 & 73.9 & 3.4 \\
2000 & 183741 & 2.7 & 16.1 & 80.9 & 0.1 \\
2010 & 255681 & 1.34 & 15.21 & 81.25 & 2.20 \\
\hline
\end{tabular}

FUENTE: CPCJ (1958); Censos Generales de Población y Vivienda 1960, 1970, 1980, 1990, 2000 y 2010 INEGI

Igualmente, la composición de la economía dio un giro importante; conforme pasaron las décadas se fue reduciendo el sector primario y proporcionalmente fue creciendo el terciario (representado fundamentalmente por el turismo). De esta manera, la población ocupada en la agricultura, la ganadería y la pesca, en muy pocas décadas representó una fracción minoritaria, inferior al 2\%, mientras que la gente ocupada en la economía de los servicios llegó a más de $80 \%$ del total.

Puerto Vallarta, como destino turístico a nivel nacional, se ubica entre los tres primeros lugares en términos de recepción de visitantes, lo que explica que el turismo sea la base de la economía local. De hecho, existe una excesiva concentración de la actividad económica en torno al turismo, puesto que cerca del 80\% de los empleos de Puerto Vallarta se relacionan con actividades vinculadas al mismo.Su economía altamente dependiente de la actividad turística la hace muy vulnerable a las posibles contingencias del sector. Éste es probablemente el motivo por el cual se ha producido un desarrollo regional irregular, con una alta concentración productiva en la región dominante (Puerto Vallarta y Bahía de Banderas), adquiriendo una ventaja de localización sobre las demás poblaciones, lo que ha intensificado las desigualdades regionales y la polarización entre la población.

De hecho, existe una fragmentación de la ciudad en dos partes: la primera es la integrada a la dinámica del turismo y la segunda es la periferia marginada, carente de dinamismo económico y conformada por áreas deprimidas, en predios irregulares con falta de servicios básicos municipales, tales como agua, luz y drenaje.

En cuanto a la calidad de vida de sus habitantes, pese a que los indicadores de bienestar del Índice de Desarrollo Humano (IDH) muestran un rango muy alto, dichos indicadores describen valores promedio sin atender a las cuestiones de dispersión y distribución de los mismos. Estos valores contrastan con la percepción de la población local, quien afirma que su calidad de vida no es tan alta, y destacan numerosas deficiencias.

\section{Impacto social del turismo en Puerto Vallarta}

Puerto Vallarta ha experimentado un significativo crecimiento demográfico durante los últimos años, alcanzando una población de 256 mil habitantes en el año 2010 (INEGI, 2010). Su extensión territorial, de más de mil kilómetros cuadrados, le permite tener abundantes recursos naturales y una densidad poblacional baja, aunque ciertamente su población urbana está altamente concentrada en torno a la cabecera municipal.

En el año 2011, más del 80\% de la población ocupada de Puerto Vallarta se desempeñaba en el sector terciario, y de este porcentaje más de la mitad trabajaba en empleos formales, considerando el total de trabajadores inscritos en el Instituto Mexicano del Seguro Social (IMSS).

De igual modo, en términos de generación de empleos, los efectos en Puerto Vallarta son evidentes; ya que emplea de forma directa a más del 30\% del personal ocupado en el municipio. Adicionalmente, el 
turismo genera de 2 a 3 empleos indirectos por cada empleo directo, con lo que el nivel de empleo derivado de esta actividad, puede rondar los 90 o 100 mil empleos en el destino de playa jalisciense (SECTUR, 2014).

Considerando una Población Ocupada (PO) que alcanza las 120 mil personas; dicha cifra indica que casi el $80 \%$ del empleo del municipio se relaciona con actividades vinculadas con el turismo (SECTUR, 2014).

Dadas sus notables condiciones socioeconómicas, Puerto Vallarta registra niveles de bienestar, en general, por encima de otros municipios del estado y muy cercanos a los niveles que registran los municipios de la ZMG. El IDH para el año 2005, es considerado alto (0.876), incluso mayor al IDH estatal (0.82) y al del resto de municipios del estado, exceptuando a Guadalajara y Zapopan. Con respecto a los componentes del IDH, Puerto Vallarta destaca muy por encima de la mayoría de municipios del estado en cuanto a esperanza de vida al nacer, alfabetismo, salud y nivel de ingreso promedio de sus habitantes (SECTUR, 2014).

En este último rubro, Puerto Vallarta sólo es superado por el municipio de Zapopan, que para el año 2005 reportó un ingreso de 16,356 dólares per cápita en paridad del poder adquisitivo (PPC), comparado con 14,987 dólares de Puerto Vallarta (SECTUR, 2014).

Si bien es cierto que Puerto Vallarta mantiene estándares de calidad de vida comparativamente altos con respecto al resto de los municipios del estado de Jalisco, aún presenta algunos rezagos en indicadores que impactan la competitividad y el bienestar de sus habitantes. Uno de ellos es el grado de conectividad, el cual se mantiene en un nivel bajo; otro es la poca diversificación de su economía, que al estar centrada en la actividad turística incrementa su vulnerabilidad ante posibles contingencias externas, dada la fuerte dependencia existente del mercado estadounidense.

\section{Impacto ambiental del turismo en Puerto Vallarta}

México cuenta con una extraordinaria diversidad biológica; sin embargo, la presión sobre los recursos naturales aumenta día con día y el efecto sobre los ecosistemas se manifiesta notablemente en la pérdida de especies y en la fragmentación de su hábitat.

Gran parte de esta diversidad se concentra en las costas de México, a lo largo de los aproximadamente $11,000 \mathrm{~km}$. de litoral, que albergan los 167 municipios costeros, en las 17 entidades federativas costeras del país.

Debido al crecimiento poblacional, así como al incremento de la inversión privada, en los últimos años se ha detectado una mayor presión ambiental en los litorales, lo que ha traído nuevas formas de ocupación y aprovechamiento de recursos naturales (PROFEPA, 2014)

El desarrollo turístico inmobiliario en las costas (construcción de infraestructura básica, hoteles, condominios, campos de golf, marinas, obras complementarias, casas habitación, residenciales, entre otros) ocasionan fuertes presiones ambientales a los ecosistemas costeros, los cuales son considerados frágiles (manglares, arrecifes, duna costera, marismas, etcétera), principalmente por la falta de una adecuada planeación y la inobservancia de la normatividad ambiental por parte de los inversionistas, ya que desarrollan sus proyectos turísticos sin contar con las autorizaciones federales ambientales (autorización de impacto ambiental y cambio de uso de suelo forestal) y patrimoniales (concesión, permiso o autorización).

Los principales impactos ambientales negativos generados por el desarrollo de infraestructura turística son:

- Modificación y destrucción del hábitat de flora y fauna terrestre y acuática.

- Cambios de uso de suelo forestal.

- Generación de residuos peligrosos.

- Contaminación de suelos y cuerpos de agua 
por emisiones líquidas (descargas de aguas residuales, aceites, lubricantes e hidrocarburos).

- Introducción de especies exóticas.

- Emisión de ruidos y vibraciones por el empleo de maquinaria pesada.

- Alteración de dunas costeras.

- Obstrucción de cuerpos de aguas superficiales y subterráneas.

- Remoción de vegetación de manglar por la apertura de caminos, zonas de tiro, bancos de explotación e instalación de campamentos y oficinas.

Desde el punto de vista patrimonial, el desarrollo turístico ha generado:

- Ocupación ilegal de la Zona Federal Marítimo Terrestre (ZOFEMAT) y los Terrenos Ganados al Mar (TGM), tanto por nacionales como por extranjeros.

- Incumplimiento a lo establecido en los instrumentos que amparan el uso, goce o aprovechamiento de la ZOFEMAT y los TGM.

- Propiedades que, por la falta de acceso, convierten a las playas públicas en privadas.

- Empresas de bienes raíces, que no orientan a sus compradores acerca de las obligaciones que establece la ley y el título de concesión con respecto de la ZOFEMAT.

De acuerdo a los datos obtenidos en el AGEB (Área Geo- estadística Básica) y en los mapas de marginación urbana elaborados por la CONAPO (Consejo Nacional de Población), se logró determinar que hasta el 2010, la población en Puerto Vallarta se encontraba distribuida de la siguiente manera:

- $32 \%$ de la población con un nivel económico alto.
- 55\% de la población con un nivel económico medio.

- $13 \%$ de la población con un nivel económico bajo.

\section{Actitud de la población receptora de Puerto Vallarta}

Si lo que se pretende es analizar la percepción que tienen las personas de Puerto Vallarta sobre el impacto de la actividad turística, es necesario entender también cuáles son las actitudes de los habitantes de la ciudad frente al turismo.

Partiendo de este hecho, se encuestó a la población de la ciudad de Puerto Vallarta para conocer cuál es su postura frente al turismo, y poder determinar en cuál de los cuadrantes de la matriz de Bjorklund y Philbrick se encuentran los habitantes de esta ciudad.

Tabla 3: Actitud de la Sociedad Vallartense.

\begin{tabular}{|lc|}
\hline \multicolumn{1}{|c|}{ Frecuencia } \\
\hline $\begin{array}{l}\text { Aceptación } \\
\text { abierta/Beneficio }\end{array}$ & 266 \\
\hline $\begin{array}{l}\text { Aceptación en } \\
\text { silencio/Beneficio }\end{array}$ & 92 \\
\hline $\begin{array}{l}\text { Rechazo abierto/Sin } \\
\text { beneficio }\end{array}$ & 15 \\
\hline $\begin{array}{l}\text { Aceptación en silencio/Sin } \\
\text { Beneficio }\end{array}$ & 383 \\
\hline \multicolumn{1}{|c|}{ Total } \\
\hline
\end{tabular}

Fuente: Elaboración propia.

Gráfica 1: Actitud de la sociedad Vallartense. 
Fuente. Elaboración Propia

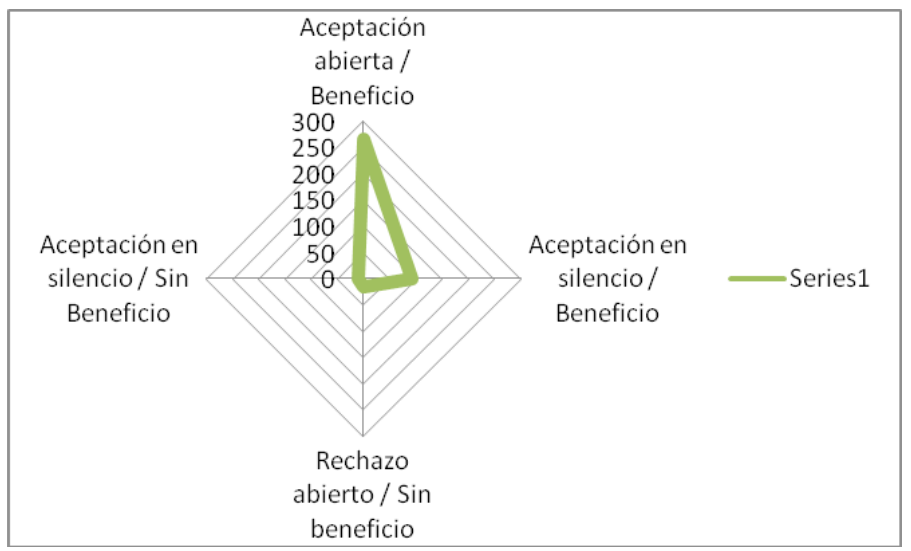

Se puede observar la tendencia de la población de Puerto Vallarta, donde en su gran mayoría aceptan la actividad turística porque les genera beneficios, ya sea económico o social.

Con los datos de la tabla y la gráfica radial, se puede entonces dibujar la matriz de Bjorklund y Philbrick, para medir la situación actual de la población de Puerto Vallarta frente al turismo, la cual quedaría de la siguiente manera:

Figura 1: Actitud/Comportamiento

Activo

Pasivo
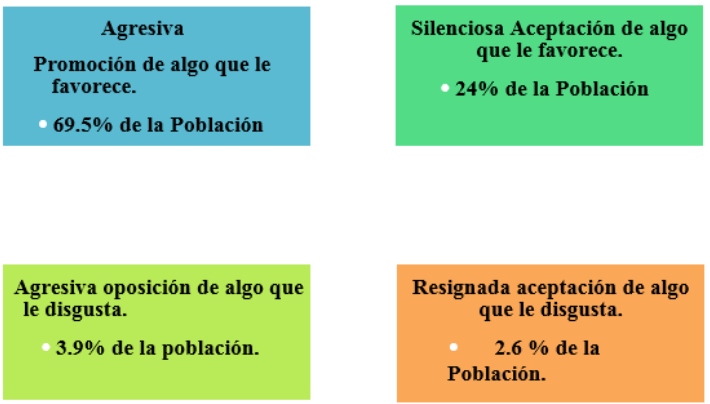

Fuente: Elaboración propia con base en el modelo de Bjorklund y Philbrick.

Es evidente que en una ciudad como Puerto Vallarta la mayoría de la población se encuentre dentro del cuadrante uno, puesto que en esta ciudad se vive del turismo. Como ya se mencionó anteriormente, aproximadamente el $85 \%$ de la población depende directamente de la actividad turística, mientras que el resto depende indirectamente.
Esto se debe a que el movimiento de recursos en la ciudad es provocado por el turismo, de tal forma de que si el turismo dejase de existir en la ciudad ésta prácticamente se iría a la quiebra.

\section{Gastronomía de Puerto Vallarta}

Las empresas de alimentos y bebidas en la ciudad de Puerto Vallarta suman un total de 1,645 según el Directorio Estadístico Nacional de Unidades Económicas (DENUE, 2017) excluyendo a los negocios que pertenecen a cadenas nacionales y/o internacionales, empresas de autoservicio, empresas que su actividad principal sea la venta de bebidas y las empresas de alimentos que se encuentren dentro de hoteles, de los cuales el $20 \%$ de estas empresas pertenecen al sector de microempresas que venden sus servicios de manera semifija en las vías públicas.

El análisis de las zonas para comprobar la posibilidad de división, comienza en analizar la forma total, buscando estrangulamientos que puedan marcar una separación natural como mojones o bordes que marquen la línea entre partes de la ciudad, una división clara en las ciudades latinoamericanas que se construyeron a la orilla del mar, y posteriormente son marcadas por una avenida costera (BOULLON, 2017: 69).

Con base al estudio que se realizó en la ciudad de Puerto Vallarta entre septiembre del 2017 y marzo de 2018 se generó una división de las zonas de Puerto Vallarta mediante la separación de las barreras que se forman de la actividad turística, se nombraron como Zona Turística, Zona de Transición y Zona Periferia; La Zona Turística que tiene una extensión de 8.84 kilómetros cuadrados contemplándose de la orilla del mar hasta la paralela Carretera Federal 200 extendiéndose por la colonia centro y llegando a la salida de la ciudad, regresando a la Carretera Federal 200 cuenta con 479 empresas de alimentos, Zona de Transición que tiene una extensión de 12.19 kilómetros cuadrados colindante de la anterior zona turística y abordando hasta la Avenida México llegando hasta el rio Pitillal y extendiéndose por la Avenida Francisco Villa, cuenta con 388 empresas de 
alimentos y la Zona Periferia que tiene una extensión de 25.76 kilómetros cuadrados que se ubica de la anterior zona hasta los límites de los que está consolidado como ciudad de Puerto Vallarta cuenta con 778 empresas de alimentos. Para explicar mejor estas divisiones de las zonas que se utilizaron en esta investigación se generó una imagen (ilustración 1).

Ilustración 1 Divisiones de las Zonas en la ciudad de Puerto Vallarta (Elaboración Propia)

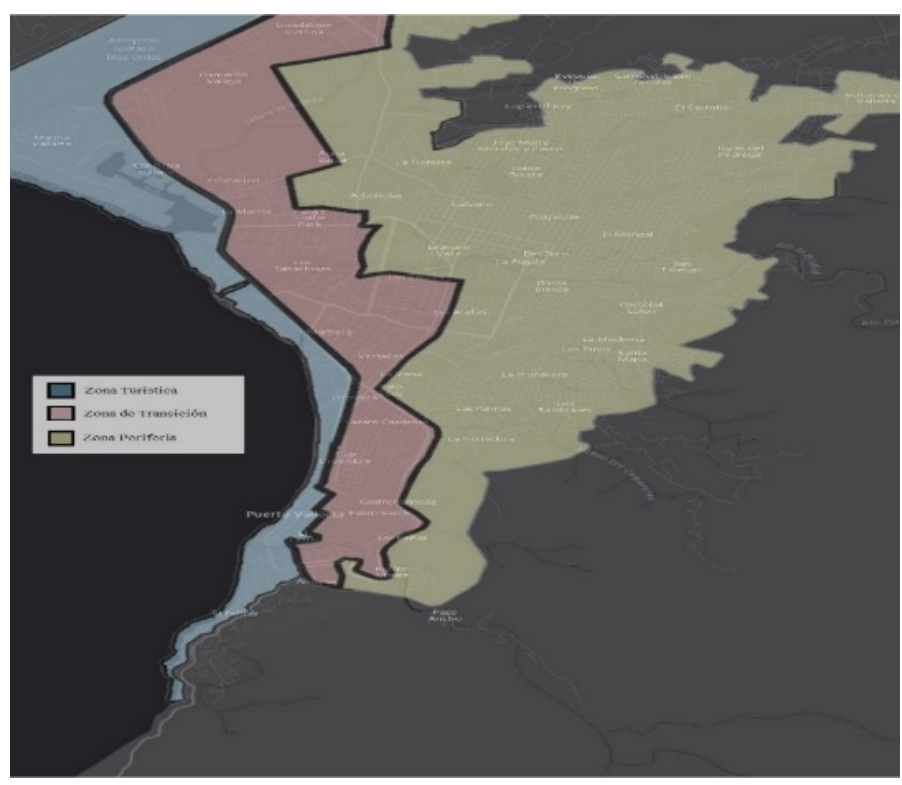

Dentro de las clasificaciones que se hace en el DENUE se ve representado un tipo de alimento que representa a la comida callejera en México y siendo el cuadro alimento icónico de la cocina mexicana en el mundo siendo el $34 \%$ del total de empresas de alimentos en la ciudad de Puerto Vallarta de la clasificación de "Tacos"; que en su mayoría son establecimientos de alimentos característicos por la venta del platillo que lleva este nombre en sus diferentes presentaciones.
Tabla 4: Clasificación de las empresas de alimentos en Puerto Vallarta.

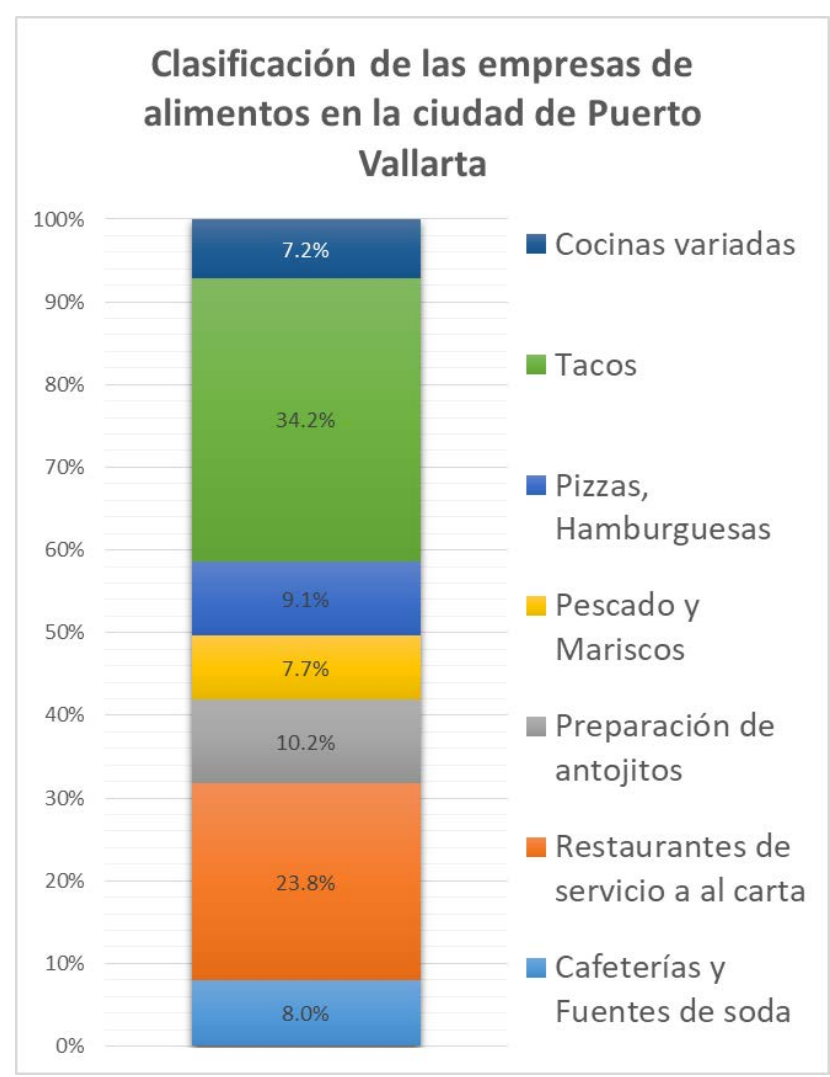

Fuente: Elaboración propia.

\section{Conclusiones}

En materia de hospitalidad en general se percibe en los habitantes de Puerto Vallarta miran con buenos ojos el desarrollo y crecimiento de la actividad turística, el $69.5 \%$ de los habitantes encuestados tienen una aceptación abierta ante esta actividad, el $24 \%$ tiene una aceptación en silencio y sólo el $2.6 \%$ manifiesta un rechazo abierto.

Esta valoración por parte de la sociedad vallartense puede entenderse tras el significado de la derrama económica que deja la actividad turística en el destino, la generación de fuentes de empleo y el constante flujo de dinero.

En materia de gastronomía las 1,645 unidades económicas del sector imprimen un valor al desarrollo del sector en el destino, tras la clasificación de las empresas de alimentos es notable el predominio de la comida callejera expresada en puestos de tacos con 
un $34.2 \%$, de establecimientos de esta índole, contra el $23.8 \%$ de establecimientos de restaurantes a la carta.

\section{Referencias}

1 BOULLON, R. Planificación del espacio turístico. México: Trillas. 2017.

2 DENUE. (2017). Directorio Estadístico Nacional de Unidades Económicas. Consultado el 27 de Enero del 2019 en www.inegi.org.mx/app/mapa/denue. Enero del 2019. 2009.

3 FONATUR. (2001). Plan Maestro de Desarrollo Urbano Turístico de Bahía de Banderas. Estados de Jalisco $y$ Nayarit. En www.sedesol.gob.mx/subsecretarias/desarrollourba no/ordonez/quienessomos.htm

4 INEGI. Jalisco, G. d. Jalisco. 2014.

5 JALISCO. Puerto Vallarta. 2014. Consultado el 27 de Enero del 2019 en www.jalisco.gob.mx/es/jalisco/municipios/puertovall arta.

6 INEGI. Instituto Nacional de Estadística, Geografía e Informática. JALISCO: 2010.

7 PROFEPA. (17 de Julio de 2014). Impacto de Desarrollos Turísticos. Consultado el 7 de Julio de 2015, de Impacto de Desarrollos Turísticos: www.profepa.gob.mx

8 SECTUR. Agendas de Competitividad de los destinos turísticos en México. Puerto Vallarta Jalisco. 2014

9 VIRGEN AGUILAR, C. R. Ciclo de Vida de un destino Turístico; CULTUR, Recuperado el 27 de 15 de enero de 2019. 2018.

10 VIRGEN AGUILAR, C. R. El turismo en Puerto Vallarta- pasado y presente. Jalisco: Trauco Editorial. 2014 a. 